\title{
Don't define nanomaterials
}

\author{
Basing regulations on a term with no scientific justification will do \\ more harm than good, argues Andrew D. Maynard.
}

$\mathrm{F}$ ive years ago, I was a proponent of a regulatory definition of engineered nanomaterials. I have changed my mind. With policy-makers looking for clear definitions on which to build 'nano-regulations', there is a growing danger of science being pushed aside.

It makes sense to assume that nanomaterials could come with unanticipated risks. A rapidly growing body of research indicates that some nanoscale materials behave differently from their bigger and smaller counterparts ${ }^{1}$. For instance, normally

benign titanium dioxide - widely

used as a whitener - becomes increasingly toxic as its particle size shrinks. Nanoscale titanium dioxide has been classified as a potential human carcinogen by the US National Institute for Occupational Safety and Health.

But it is becoming clear that many parameters other than size modulate risk, including particle shape, porosity, surface area and chemistry. Some of these parameters become more relevant at smaller scales - but not always. The transition from 'conventional' to 'unconventional' behaviour, when it does occur, depends critically on the particular material and the context.

A 'one size fits all' definition of nanomaterials will fail to capture what is important for addressing risk.

\section{TERM OF ART OR SCIENCE?}

Concern over the risks of engineered nanomaterials has led organizations around the world to reconsider their regulations. In June, the US Food and Drug Administration issued draft guidance to help companies determine whether their products utilize nanomaterials. The US Environmental Protection Agency has just issued draft guidelines for when a nanomaterial should be considered a 'new' substance under the Federal Insecticide, Fungicide and Rodenticide Act, even if a largerscale version is already in use.

In Europe, the REACH regulations (on the registration, evaluation, authorization and restriction of chemicals), in force since 2007, have been accompanied by contentious (and so far unresolved) discussions about how they apply to engineered nanomaterials. New European cosmetics regulations require ingredients that are engineered nanomaterials to be listed on product labels by 2013 .

Many argue, and I once agreed, that a definition of engineered nanomaterials is needed to ensure their safe use under such regulations. But a sensible definition has proved hard, if not impossible, to arrive at.

Last year, the European Commission (EC) released this draft definition for public comment: "a material that consists of particles with one or more external dimensions in the size range $1 \mathrm{~nm}-100 \mathrm{~nm}$ for more than $1 \%$ of their number"; and/or "has internal or surface structures in one or more dimensions in the size range $1 \mathrm{~nm}-100 \mathrm{~nm}$ "; and/or

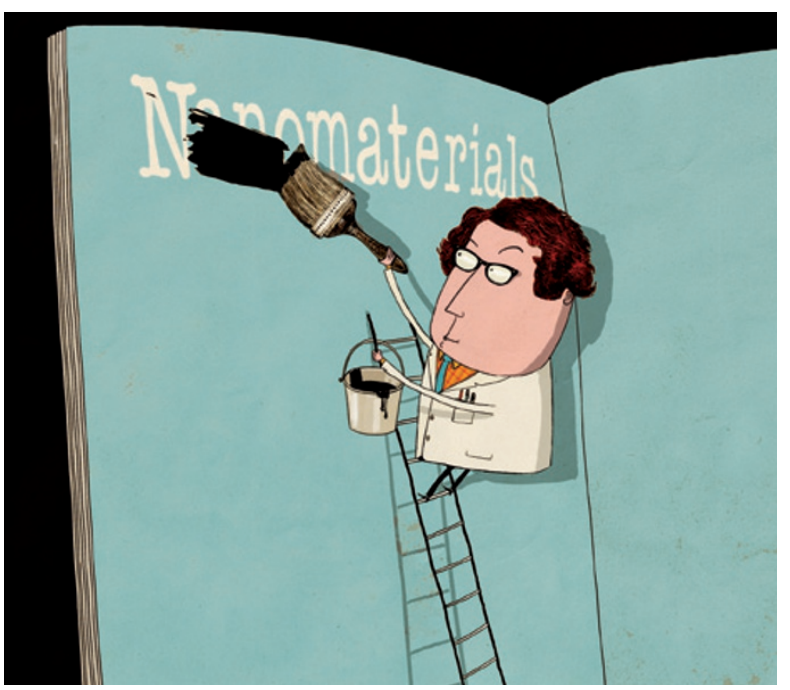

"has a specific surface area by volume greater than $60 \mathrm{~m}^{2} \mathrm{~cm}^{-3}$, excluding materials consisting of particles with a size lower than $1 \mathrm{~nm}$ ". Despite a stated intent to base the definition on "available scientific knowledge", none of these criteria is supported by current data on nanomaterial risk.

Unsurprisingly, the commission has not yet been able to reach a consensus on a definition. The coordinator of the EC's nano team has been quoted as saying that ultimately the decision on a regulatory definition of nanomaterials would be a "policy one". This should ring alarm bells throughout the scientific community: it implies that the basis for nanomaterial regulation will be a 'term of art' rather than science.

Strict definitions of harmful materials have caused problems before. The mineral Libby vermiculite, for instance, contains deadly asbestiform fibres - and yet it slipped through the regulatory net for many years because it didn't fit the official definition of asbestos.
To avoid similar problems with nanomaterials, regulators should work with a list of nine or ten attributes (including size and surface area) for which certain values trigger action. This list of trigger points would differ from the EC black-and-white definition in several key ways: attributes other than size and surface area would be included; cut-off values would be compound-specific and based on current science; and the criteria would allow for changes in a given material over time. Such regulatory sophistication will obviously take a lot of work. Much more research is needed to pin down how much a material would need to change to trigger a regulatory red flag: should a $1 \%$ change in mean particle size of a material previously determined to be safe, say, raise concerns, or should it be a $50 \%$ change? But enough is known today for an expert panel to begin determining key attributes and preliminary trigger points for many materials.

These trigger points must be flexible, so that they can be modified as evidence grows. Adaptive regulations are necessary to respond to scientific evidence, although current US legal mechanisms make them hard to enact.

Last month, the US Executive Office of the President released policy principles that stress the need for science-based regulation of engineered nanomaterials, stating: "A focus on novel properties and phenomena observed in nanomaterials may ultimately be more useful than a categorical definition based on size alone." 2 . This is an important step in the right direction. Even so, assuming that nanomaterials are a unique class of material continues to present a stumbling block to effective regulation.

Andrew D. Maynard is director of the Risk Science Center at the University of Michigan School of Public Health, Ann Arbor, Michigan 48109-2029, USA.

e-mail:maynarda@umich.edu

1. Maynard, A. D., Warheit, D. \& Philbert, M. A. Toxicol. Sci. 120, S109-S129 (2011).

2. Holdren, J. P., Sunstein, C. R. \& Siddiqui, I. A. Policy Principles for the U.S. Decisionmaking Concerning Regulation and Oversight of Applications of Nanotechnology and Nanomaterials (Executive Office of the President, 2011).

Further reading accompanies this article online at go.nature.com/xrp2jp 